\title{
APLICACIÓN DEL CUESTIONARIO \\ “POSITIVE_PERSONAL_TOOL” Y SU RELACIÓN CON LOS MIEDOS ADOLESCENTES
}

\author{
Cristina Agabo García \\ Facultad de Ciencias. Universidad de Cádiz \\ cristina.agabo@uca.es
}

Recepción Artículo: 13 octubre 2021

Admisión Evaluación: 13 octubre 2021

Informe Evaluador 1: 13 octubre 2021

Informe Evaluador 2: 14 octubre 2021

Aprobación Publicación: 14 octubre 2021

\section{RESUMEN}

La era postmoderna actual junto con la pandemia de la COVID-19 han provocado una situación de incertidumbre constante que puede influir en el desarrollo personal de los adolescentes. Por ello, es importante reforzar las capacidades (sociales, emocionales, morales y cognitivas) de los alumnos de forma que se incentive un desarrollo positivo personal. En este trabajo, se ha diseñado y validado un test multidimensional para evaluar las áreas de desarrollo positivo adolescente y se ha determinado la relación de éstas con el afrontamiento de los diferentes miedos adolescentes. El test denominado "Positive_Personal_Tool" se realizó mediante el software "EasyLMS" en una muestra de 147 alumnos de dos institutos de la provincia de Cádiz (España). Además, se realizaron el test de Rosenberg (1965) de Autoestima para validar el test diseñado y el test de Satisfacción por la vida de Huebner (1991) como test control. Los resultados obtenidos mostraron que el test creado es fiable (alfa de Cronbach = 0,7-0,9) para cada grupo de variables evaluadas (miedos, áreas y capacidades), sin embargo, su correlación con la autoestima se vio afectada por el sesgo poblacional con autoestima inflada, por lo que habría que tener en cuenta otros activos del eje del desarrollo personal positivo. De forma general se obtuvo que el $50 \%$ de los alumnos mostraron una autoestima media y el 20\% presentó una satisfacción por la vida baja y las diferentes áreas personales puntuaron por debajo de la media (en torno al 40\%) sobre todo las capacidades de creatividad, toma de decisiones, optimismo y tolerancia a la frustración. Esto junto con los valores atípicos obtenidos del miedo a lo desconocido remarcan el efecto que ha tenido la pandemia provocada por la COVID-19 en el desarrollo personal adolescente.

Palabras clave: miedos adolescentes; áreas de desarrollo personal; test multidimensional de autoconcepto; autoestima 


\begin{abstract}
Design, application and validation of the survey named as "POSITIVE_PERSO-NAL_TOOL"; evaluating the positive personal development areas and its relathioship with teenegers fears. The current postmodern era coupled with the COVID-19 pandemic have provoked a constant situation of fear and uncertainty that could influence the personal development of adolescents. Therefore, it is important to strengthen the capacities (social, emotional, moral and cognitive) of the students in a way that encourages a positive personal development. In this work, a multidimensional test has been designed to evaluate the personal positive development of teenegers. It has been tested if this test was valid and if the results obtained were mathematically related to the different adolescent fears. The test called "Positive_Personal_Tool" was performed using the "EasyLMS" software on a sample of 147 students in high School from Cádiz region in Spain. The Rosenberg test (1965) for self-steem evaluation and Huebner test (1991) for Life Satisfaction were also used as validation and control tests respectively. The results obtained showed that the created test was reliable given that Cronbach's alpha (0.7-0.9) for each studied variable (fears, areas and capacities). However, the correlation between the PPTT test and selfesteem was affected by population with excess of self-steem. So, other actives of positive personal development should be taken into account for future complete validation of this test. Thus, in general, it was obtained $50 \%$ of medium level of self-esteem and $20 \%$ of low level of life satisfaction. The different personal areas scored below the average (around 40\%) due to, above all, capacities such as the creativity and decision-making capacities, optimism and tolerance to frustration. This fact, joined to very atypical values of fear of the unknown situations; highlighted the effect of COVID-19 pandemic to personal positive development of teenegers.
\end{abstract}

Keywords: teeneagers fears; personal development areas; self-concept multidimensional test; self-esteem

\title{
INTRODUCCIÓN
}

Las competencias o elementos claves del desarrollo positivo adolescente se definen como la forma en que los adolescentes utilizan algunas de sus habilidades, valores y conocimientos para resolver de forma adecuada una determinada tarea en un contexto determinado. Dado la situación actual de incertidumbre constante que nos impone la era postmoderna actual (y la que ha desarrollado la pandemia del COVID-19 en particular) nos encontramos en una vida gobernada por continuas sobrecargas de trabajo, incertidumbre, miedo, ansiedad, etc. Este contexto es, por tanto, idóneo para el desarrollo potencial del sufrimiento psíquico en la población general, incluidos niños y adolescentes, que puede durar un período de tiempo significativo después de la pandemia (Huremovic, 2019), convirtiéndose en una barrera adaptativa e incluso desarrollar un trastorno posterior que altere seriamente su desarrollo (Pérez Grande, 2000). Previamente a la pandemia, diferentes investigaciones han surgido acerca de los miedos en niños y adolescentes y en cómo un desarrollo excesivo de los mismos puede derivar en consecuencias psicopatológicas. Méndez, Inglés, Hidalgo, García-Fernández y Quiles (2003) determinaron que los miedos adolescentes (MA) más comunes fueron los miedos al contacto físico, muerte y miedo sociales y mostraron interacciones género-edad estadísticamente significativas en miedos sociales en general, así como en miedo a la autoridad, a la muerte, a la evaluación del rendimiento y a lo desconocido. Por ello, la definición de los diferentes elementos que definen un desarrollo positivo adolescente y su evaluación sería una herramienta valiosa para poder incrementar sus recursos y así presentar un desarrollo más saludable que le otorgue la capacidad de superar situaciones de incertidumbre o miedo como la que se está viviendo en nuestros días, haciéndolos más resistente a factores de riesgo como los trastornos depresivos o el miedo crónico, etc (Benson, Mannes, Pittman, y Ferber, 2004).

Diferentes modelos de desarrollo positivo han sido enunciados a lo largo de los últimos años como modelos complementarios al modelo del déficit (Lerner et al., 2005; Roth y Brooks-Gunn, 2003). La confluencia de todos ellos ha dado como resultado un modelo basado en competencias cognitivas, conductuales y sociales y además, contribución (a sí mismo, a la familia, a la comunidad y a la sociedad civil) añadiendo un componente ideológico.

Bajo este contexto, Oliva et al., (2008) enunciaron un modelo de desarrollo positivo adolescente a partir de 
las opiniones de un grupo de expertos sobre las competencias que engloban el desarrollo positivo. Se obtuvieron un listado de características incluyendo no sólo competencias específicas, sino también valores, capacidades 0 habilidades, cuya combinación cualitativa obtiene rasgos de personalidad, destrezas, valores y conocimientos que posibilitan el desarrollo personal del adolescente en la sociedad actual. Todas estas características se englobaron en cuatro áreas (ADPAs): social, moral, emocional y cognitiva. Las competencias que definen el desarrollo personal (Autoestima, autoconcepto, autoeficacia, autoeficacia, autocontrol, autonomía personal, sentido de la pertenencia e iniciativa) se nutren de los diferentes ADPAs. Oliva et al., (2008) además afirmaron que estas competencias también se relacionan y potencian unas a otras, de modo que el desarrollo personal tiene que ver con las influencias dinámicas y recíprocas que se establecen entre las competencias específicas incluidas en cada uno de los grupos. Por ello, establecer relaciones entre los diferentes grupos sería un potente indicador para obtener previsiones.

Desde hace algunas décadas se ha venido subrayando la importancia del Autoconcepto/autoestima en el bienestar psicosocial desde diferentes ámbitos de la Psicología como el ámbito educativo, social o cognitivo (Fuentes, García, García y Lila, 2011). Sin embargo, los términos autoconcepto y autoestima no tienen una delimitación clara dado su uso indistinto para referirnos al conocimiento que elabora un individuo sobre sí mismo. Algunos autores afirman que sí existe una sutil diferencia entre ambos conceptos (Musitu, Román y García, 1988; Rosenberg, 1965). Por ello, la forma de evaluar ambos conceptos en individuos en general y en adolescentes en particular es diferente. A pesar de que algunos autores usan tests de evaluación de autoconcepto unidimensionales (Villar, Michael y Gribbons, 1995); otros autores afirman que ambos constructos son multidimensionales (Fernández-Zabala, Goñi, Rodríguez-Fernández, Goñi, 2015; Piers y Harris (1969); García y Musitu, (2014). Sin embargo, ninguna de sus concepciones, coinciden con el modelo de desarrollo positivo propuesto por Oliva et al., (2008).

El estudio del autoconcepto y la autoestima desde su comienzo se ha realizado con el fin de relacionarse con otras variables psicológicas como la edad (Góngora, Fernández y Castro, 2012); el género (Padilla, García y Suárez, 2010); el nivel socioeconómico (Gordillo, Castro, Herrera, Acuñas y Solanes, 2011) 0 el rendimiento escolar (Rodríguez, Droguett y Revuelta, 2012). Pero existen muy pocos estudios de su relación con el afrontamiento de los MA (Byrne, 2000; Beltrán Martínez, 2014).

Además, la elaboración de dichos modelos hasta la actualidad ha experimentado cambios notables no sólo en el número de contenidos, sino en la diferenciación y organización de los mismos hasta llegar a formar una teoría integrada y coherente (Serrano-Muñoz, 2014). La revisión de los instrumentos de evaluación de la autoestima y el autoconcepto consultados en la literatura especializada ha llevado a la necesidad del planteamiento de dos preguntas principales: ¿Es posible elaborar un cuestionario para la evaluación del autoconcepto en adolescente partiendo de un modelo multidimensional basado en el modelo de las ADPAs? Además, bajo el contexto de miedo permanente, ¿qué relación tienen las diferentes ADPAs con el afrontamiento de los MA?

\section{OBJETIVOS DE LA INVESTIGACIÓN}

Los objetivos a cumplir mediante este trabajo son:

Conocer el estado psicosocial general de los alumnos encuestados tras la pandemia

Conocer por parte de los alumnos, los distintos ámbitos que conforman el desarrollo personal para identificar sus debilidades y fortalezas

Proponer un nuevo test para evaluar las ADPAs

Entrenar la autorregulación del miedo en el contexto actual de pandemia.

Determinar si existe relación significativa entre las ADPAs y el afrontamiento de MA. 


\section{MUESTRA Y/O PARTICIPANTES}

\section{Participantes}

Participaron en esta investigación un total de 147 estudiantes de la provincia de Cádiz de dos Institutos IES Santo Domingo de El puerto de Santa María y el IES Padre Luis Coloma de Jerez de la Frontera de los cursos de $3^{0}$ y $4^{0}$ de la E.S.0: 93 féminas (\%66,3) y 55 varones (\%36,7). Se les aseguró, por otro lado, el anonimato de las respuestas, así como la participación totalmente voluntaria en la investigación. A pesar de que lo habitual en la determinación de la consistencia interna de una escala se necesiten entre cinco y veinte participantes por cada ítem; se acepta que por lo general se realice con una muestra de 100 participantes (Campo-Arias y Oviedo, 2008).

\section{METODOLOGía Y/O INSTRUMENTOS UTILIZADOS}

\section{Instrumentos}

\section{Test: "Personal Positive Tool"}

Para el estudio indicado se ha diseñado un cuestionario al que hemos denominado: "Personal_Positive_tool test (PPTT)" ya que dará cuenta al alumno de sus activos y herramientas que posee para la superación de situaciones de miedo. Se seleccionaron lo MA más comunes en la sociedad adolescente (Méndez et al., 2003; Zubeidat, Salinas y Sierra, 2007) y se englobaron en tres conjuntos de MA: 1.Falta de afecto/soledad; 2. Miedo a lo desconocido y 3 . Miedo a la evaluación negativa. A partir de dichos conjuntos de MA se han elaborado las preguntas del cuestionario denominado PPTT. Estas capacidades se han elaborado en base a las capacidades descritas por Oliva et al., (2008) que determinan el desarrollo positivo personal adolescente (Tabla 1) y que se engloban en diferentes ADPAs.

Tabla 1. ADPAs, capacidades a autoevaluarse y opción de respuesta correspondiente.

\begin{tabular}{lll}
$\begin{array}{l}\text { Ámbito o categoría del área } \\
\text { de desarrollo personal }\end{array}$ & Ítem a autoevaluar & $\begin{array}{l}\text { Respuesta } \\
\text { seleccionada }\end{array}$ \\
\hline Social & 1. Asertividad & A. \\
& $\begin{array}{l}\text { 2. Habilidades relacionales } \\
\text { 3. Habilidades para la resolución de } \\
\text { conflictos interpersonales }\end{array}$ & B. \\
& 4. Habilidades comunicativas & D. \\
& 5. Otra & E. \\
& \multicolumn{1}{c}{ Cocial- } \\
& $\begin{array}{ll}\text { 1. Compromiso } \\
\text { Prosociabilidad }\end{array}$ & A. \\
& 2. Responsabilidad & B. \\
3. Justicia & C. \\
& 4. Igualdad-diversidad & D. \\
& 5. Otra & E.
\end{tabular}

Emocional

1. Empatía-emociones de los demás

A.

3. Conocimiento y manejo de las propias emociones

B.

4. Tolerancia a la frustración

C.

5. Optimismo y sentido del humor

D.

5. Otra

E. 
Cognitiva

1. Capacidad de análisis críticopensamiento critico

A.

2. Creatividad

B.

3. Capcidad de planificación y revisión

C.

4. Capacidad para tomar decisiones

D.

5. Otra

E.

El total de preguntas del test fue de 36 preguntas. Cada pregunta tendrá 5 respuestas posibles pudiéndose marcar todas las que se quiera (por lo que es multirrespuesta) de forma que cada respuesta seleccionada puntuará como 1 para cada una de las capacidades de desarrollo personal del alumno excepto de la respuesta denominada "Otra" que puntuará como 0.

\section{Test de Validación y test control}

Para la validación del PPT se usó el test de autoestima mono-dimesional de Rosenberg (1965). Como test control se utilizó la escala unidimensional para la evaluación de la satisfacción vital de Huebner (1991). Este test se aplicó dado que el concepto de satisfacción con la vida es una evaluación global y positiva de la calidad de su vida y contribuye a la prevención, detección precoz e intervención en diversas poblaciones en condiciones de riesgo (Fernández-Zabala et al., 2015). Estos cuestionarios poseen escalas tipo Likert.

\section{Metodología}

Para el desarrollo del test por parte de los alumnos se utilizó un software denominado "EasyLMS" de licencia libre durante siete días en la página https://www.onlinequizcreator.com/es/. Se realizó de forma grupal en una sesión de tutoría de los dos institutos durante la misma semana. Este tipo de softwares, no sólo son atractivos para el alumno, sino que además permiten el ahorro de tiempo en la transposición de datos a soportes informáticos y devuelven una respuesta inmediata al alumno y encuestador de los resultados. El tiempo medio de respuesta a los tres cuestionarios fue de $20 \pm 6$ minutos.

Con el propósito de comprobar las características psicométricas del PPTT se analizó la capacidad discriminativa de las capacidades usadas y se calculó la fiabilidad de los tests usando SPSS. Además, se realizó el cálculo de las correlaciones de las puntuaciones de las diferentes ADPAs con las puntuaciones a la escala de autoestima y las puntuaciones de los grupos de miedo usando el programa de Office Excel.

\section{RESULTADOS ALCANZADOS}

\section{Resultados globales de los diferentes tests}

Los resultados globales obtenidos para el test de autoestima determinaron que el 49,0\% de los alumnos mostraron una autoestima alta y el $51,0 \%$ de los alumnos poseían una autoestima media; siendo la media de puntuaciones femeninas de 28,8 \pm 6.04 (en el rango de autoestima media) y la masculina fue $31,1 \pm 4,8$ (autoestima alta). En cuanto a los resultados del test de satisfacción por la vida se obtuvo que el 19,6\% tenían una satisfacción vital baja y el $54,0 \%$ y el $26,4 \%$ una satisfacción vital media y alta, respectivamente. Por tanto, sería interesante trabajar ambos constructos en las aulas.

En el caso del PPTT Ios resultados globales obtuvieron una media de competencias de 40,8\% repartidas en diferentes áreas de forma similar (Figura 1). 


\section{APLICACIÓN DEL CUESTIONARIO "POSITIVE_PERSONAL_TOOL” Y SU RELACIÓN CON LOS MIEDOS ADOLESCENTES}

Figura 1. Valores medios obtenidos de las diferentes ADPAs

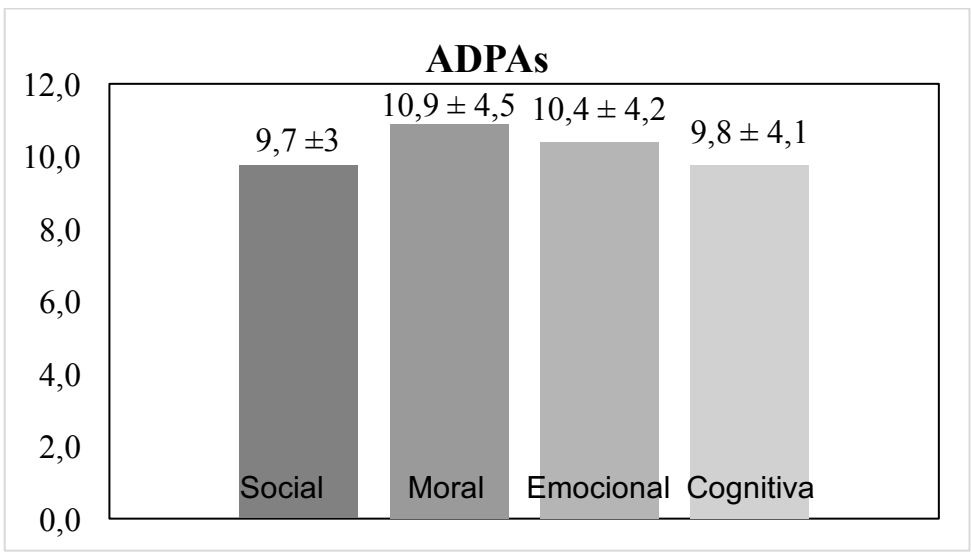

En la Figura 2, se muestra el porcentaje del promedio obtenido en las diferentes capacidades que determinan las diferentes ADPAs. Como puede observarse, Ios activos denominados como "creatividad" y "tolerancia a la frustración" son los menos representados (en torno al 4\%) del total de las capacidades de las ADPAs y las capacidades denominadas como "habilidades relacionales" "empatía", "compromiso social-prosociabilidad" son las que más representación obtienen en la muestra usada con un porcentaje promedio del doble (8\%) aproximadamente.

Figura 2. Porcentaje de los promedios obtenidos de las diferentes capacidades que describen las ADPAs.

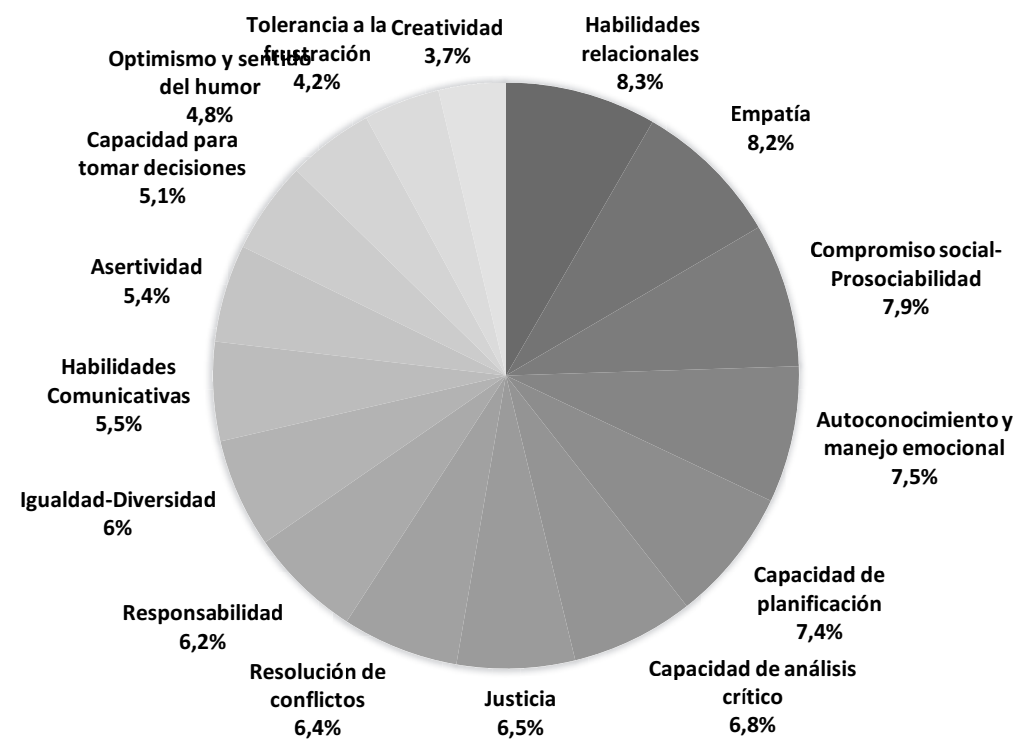


Evaluando de forma global el nivel de afrontamiento a los tres grupos de miedo se obtuvieron valores de 14,2 \pm 4 , puntos lo que corresponde un $39.6 \%$ del total (108) de los posibles puntos a obtener; resultados muy parecidos a los obtenidos en las ADPAs. Estos resultados nos dicen que deben reforzarse las capacidades en general para mejorar el enfrentamiento de los tres grupos de MA.

Además, se presentan en la Tabla 2 los datos obtenidos de las diferentes características psicométricas de cada una de las capacidades que definen la personalidad del alumno. La media se sitúa en 2,44 $\pm 0,74$, siendo las "habilidades relacionales" y la "empatía" las dos capacidades más puntuadas y los activos con menos puntuación "creatividad" y "optimismo".

Tabla 2. Parámetros estadísticos descriptivos de los diferentes activos.

\begin{tabular}{|c|c|c|c|c|}
\hline Capacidades & Media & $\begin{array}{l}\text { Desviación } \\
\text { estándar }\end{array}$ & Asimetría & $\begin{array}{l}\text { Exceso de } \\
\text { curtosis }\end{array}$ \\
\hline 1.Asertividad & 2,19 & 1,41 & 0,535 & 0,457 \\
\hline $\begin{array}{l}2 . \quad \text { Habilidades } \\
\text { relacionales* }\end{array}$ & 3,65 & 1,58 & $-0,158$ & $-0,519$ \\
\hline $\begin{array}{l}\text { 3.Resolución } \\
\text { conflictos* }\end{array}$ & 2,79 & 1,55 & 0,317 & $-0,306$ \\
\hline $\begin{array}{l}\text { 4.Habilidades } \\
\text { comunicativas }\end{array}$ & 2,23 & 1,26 & 0,106 & $-0,491$ \\
\hline 5.Prosociabilidad* & 3,34 & 1,92 & 0,175 & $-0,633$ \\
\hline 6.Responsabilidad* & 2,76 & 1,47 & 0,209 & $-0,511$ \\
\hline 7.Justicia* & 2,89 & 1,49 & 0,292 & $-0,245$ \\
\hline 8.Igualdad & 2,34 & 1,48 & 0,504 & $-0,083$ \\
\hline 9.Empatía* & 3,40 & 1,88 & 0,167 & $-0,445$ \\
\hline $\begin{array}{l}\text { 10.Manejo } \\
\text { emocional* }\end{array}$ & 3,32 & 1,57 & 0,068 & $-0,332$ \\
\hline $\begin{array}{l}\text { 11. Tolerancia a la } \\
\text { frustración }\end{array}$ & 1,94 & 1,41 & 0,921 & 1,764 \\
\hline 12.Optimismo & 1,71 & 1,30 & 0,707 & 0,007 \\
\hline 13.Análisis crítico* & 3,07 & 1,66 & 0,181 & $-0,395$ \\
\hline 14.Creatividad & 1,52 & 1,14 & 0,787 & 0,762 \\
\hline 15.Planificación* & 3,23 & 1,71 & 0,154 & $-0,362$ \\
\hline 16.Toma decisiones & 2,37 & 1,41 & 0,780 & 0,941 \\
\hline
\end{tabular}

* Activos que se sitúan por encima de la media.

La mayoría de las variables tienen asimetría >0 pero de valores bajos entre $0-0,5$ por lo que se acercan bastante a la distribución normal (Curtosis $=[-0,5 ; 0,5]$ ).

Sin embargo, las capacidades de "creatividad" y "toma de decisiones" y "optimismo" y "tolerancia a la frustración" se alejan en más de un 0,7 por lo que la distribución de los datos a la derecha de la media es más dispersa, obteniéndose distribuciones leptocúrticas. Esto indica grandes valores atípicos (valores muy bajos y muy altos).

Estos resultados se pueden apoyar en los estudios obtenidos por Gómez-Becerra, Contreras, Romero, López y Torres (2020) y Berasategui Sancho (2020) en los que se concluye que la faceta más afectada por la COVID-19 es el aspecto emocional y en concreto la facilidad a enfadarse y la tendencia a la tristeza.

En cuanto a las ADPAs (Tabla 3), presentan medias y desviaciones estándares muy parecidas y son bastante 
simétricas y su nivel de apuntamiento es bajo $(<0,5$, con excepción del área emocional que tiene una asimetría ligeramente superior a 0,5 ) por lo que se aproximan a ser distribuciones normales.

Tabla 3. Estadísticos descriptivos de las diferentes variables que describen las ADPAs de este estudio.

\begin{tabular}{lllll}
\hline Áreas/Miedos & Media & $\begin{array}{l}\text { Desviación } \\
\text { estándar }\end{array}$ & Asimetría & $\begin{array}{l}\text { Exceso } \\
\text { Curtosis }\end{array}$ \\
\hline Social & 10,5 & 3,17 & 0,394 & 0,195 \\
Moral & 11,5 & 3,41 & 0,330 & 0,024 \\
Emocional & 10,9 & 3,32 & 0,557 & 0,510 \\
Cognitiva & 10,3 & 3,45 & 0,244 & 0,173 \\
\hline Miedo 1 & 13,3 & 4,24 & 1,259 & 2,72 \\
Miedo 2 & 14,8 & 5,53 & 2,048 & 7,8 \\
Miedo 3 & 14,7 & 5,30 & 0,316 & 0,721 \\
\hline
\end{tabular}

En el caso del afrontamiento de MA (Tabla 3) se puede ver que la media y la desviación típica es parecida sin embargo la asimetría es positiva en el caso del Miedo 1 (Soledad) y Miedo 2 (Desconocido) por lo que los valores que están por encima de la media son más dispersos que los que están por debajo. Además, el exceso de curtosis, del miedo 1 y sobre todo del miedo 2 nos da cuenta que hay valores muy atípicos del afrontamiento al miedo a lo desconocido en esta distribución probablemente debido a la incertidumbre que ha existido y existe durante la pandemia de la COVID-19.

Además, el alfa de Cronbach de los tests control y validación fueron de 0,86 y 0,85 respectivamente. La consistencia interna se considera aceptable cuando se encuentra entre 0,70 y 0,90 . Sin embargo en el caso del test, propuesto, el cálculo de fiabilidad de Cronbach para cada capacidad obtuvo una fiabilidad de 0,72, para las diferentes ADPAs, el test de fiabilidad obtuvo 0,90 y desde el punto de vista del afrontamiento de los diferentes MA fue de 0,82. Éstos valores se pueden considerar aceptable teniendo en cuenta la gran cantidad de ítems que presenta nuestro test.

\section{DISCUSIÓN}

Dado estos resultados se puede decir que los alumnos presentan carencias en sus activos personales (en aproximadamente un 59,2\%) para un desarrollo positivo, siendo posiblemente la causa de obtener una autoestima media y una satisfacción por la vida mediocre. Estos resultados muestran que si bien, los alumnos muestran de forma general un balance de las diferentes ADPAs (Figura 3; 10,2 $\pm 3,9 \%$ ) lo que hace un total en ambos casos de 40-42\%; deberían trabajarse más todas ellas para poder alcanzarse el valor recomendable (60\%) según Carretero Dios y Pérez, (2007). Esto supondría aumentar, aproximadamente, en un 5\% la puntuación de las ADPAs. Estos valores bajos pueden ser fruto de la presencia de pre-adolescentes todavía en estos niveles (sobre todo en $3^{0}$ de E.S.0) cuyo estadío biológico propicia aún un incompleto desarrollo moral e ideológico, así como a la identidad sexual y desarrollo cognitivo. Sin embargo, la situación de confinamiento derivada de la pandemia por la COVID-19 también ha podido influir en el desarrollo anómalo de las diferentes ADPAs.

Como puede verse en la Figura 3, Ios resultados obtenidos muestran una correlación positiva entre autoestima y los resultados del PPTT con un $\mathrm{R}^{2}$ de 0,90 en el caso del género femenino. Sin embargo, en el género masculino no correlacionan $\left(R^{2}=0,28\right)$. 
Figura 3. Correlación de puntuación total del test de autoestima con el test PPTT en la muestra femenina.

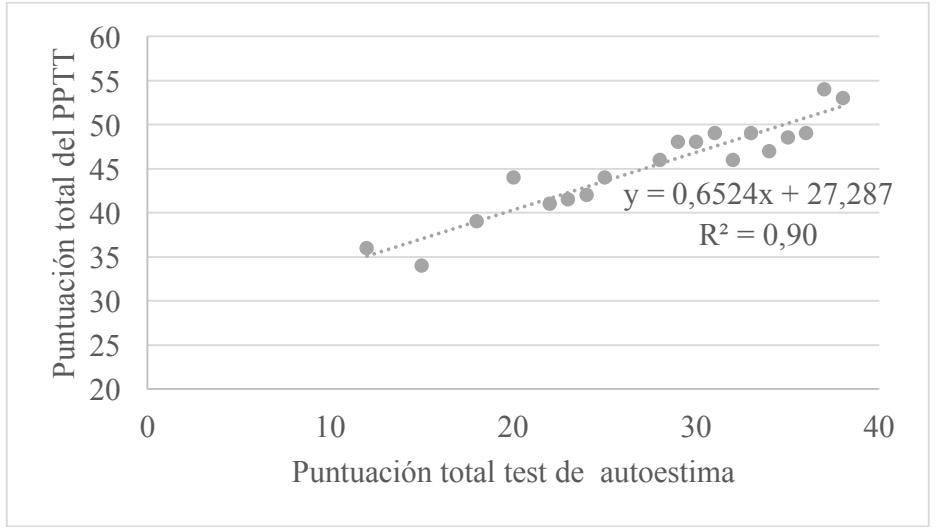

Siguiendo esta correlación se podría determinar los rangos de PPTT bajos (0-43) medios, (44-46) 0 altos (47-108) equiparándolos con los del test de autoestima de Rosenberg (1965).

Estos resultados se corresponden con aquellos obtenidos por Bleidorn et al., (2016) donde los hombres y mujeres aumentan su autoestima con la edad, aunque en los hombres en menor medida ya que desde la adolescencia poseen niveles de autoestima elevados probablemente debido a aspectos culturales que orientan a la sobre-elevación de la autoestima masculina (estereotipos de género) (Tapia, 2016).

Figura 4. Correlación de puntuación total del test de autoestima con el test PPT en la muestra masculina.

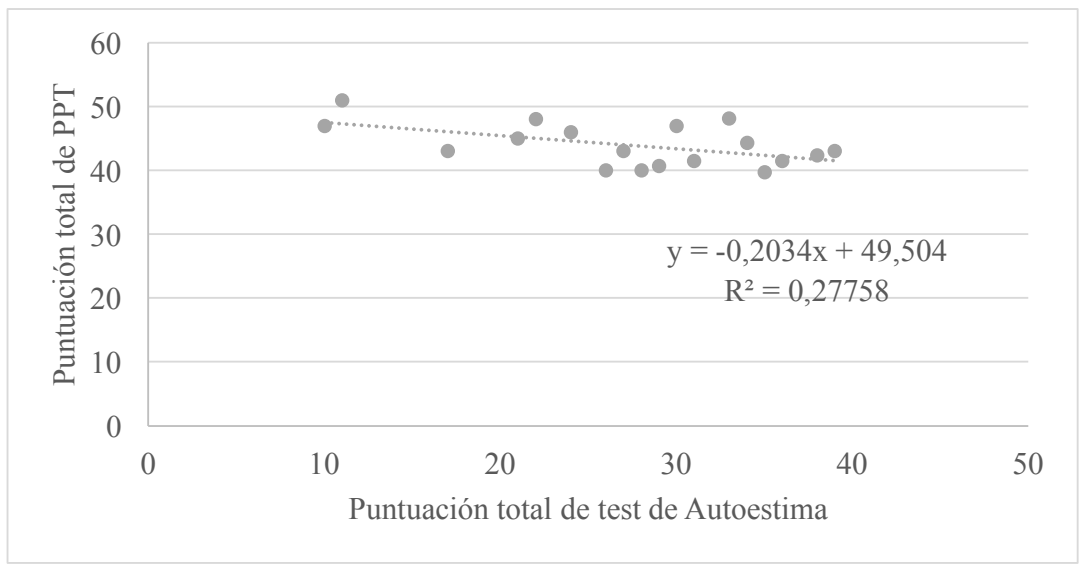

Esto hace que la correlación entre autoestima y autoconcepto sea diferente dependiendo del género debido a la estrecha diferenciación de ambos conceptos y los estereotipos. El estereotipo masculino eleva la autoestima de los jóvenes varones independientemente de las capacidades que posea.

Además, en el test de satisfacción por la vida de Huebner (1991), se puede observar que, los resultados de satisfacción por la vida alta, puntúa en un 22,2\% para el género femenino y un 30,5\% para el masculino lo que 
era de esperar teniendo en cuenta que los varones presentan un mayor nivel de autoestima.

Pero además si se correlaciona este test control, con ambos test se puede comprobar que tienen una correlación positiva para las puntuaciones de satisfacción por la vida y el test diseñado PPTT $\left(R^{2}=0,95\right)$ y una nocorrelación con la puntuación del test de autoestima tal cual. Sin embargo, si se separan por fracciones: autoestima alta y autoestima media-baja se obtienen correlaciones logarítmicas con alta bondad de ajuste $\left(R^{2}=0,95\right.$ 0,96; Tabla 4).

Dada la pre-evaluación favorable del test por tres expertos y las correlaciones parciales encontradas con el test de Autoestima de Rosenberg así como los índices de fiabilidad se podría aceptar el cuestionario basado en las ADPAs creado como herramienta para la evaluación del autoconcepto en adolescentes. Sin embargo, sería interesante realizar un test paralelo incluyendo otros activos del desarrollo positivo personal adolescente según Oliva et al., (2008) 0 usar un test de autoconcepto multi-variable como método de validación más fiable que el test de autoestima mono-variable.

Tabla 4. Mejores ajustes obtenidos tras correlacionar las puntuaciones obtenidas del test de satisfacción por la vida con los test de autoestima y PPTT realizados.

\begin{tabular}{lcc}
\hline Variables relacionadas & Ecuación & $\mathrm{R}^{2}$ \\
\hline $\begin{array}{l}\mathrm{X} \text { : Satisfacción por la } \\
\text { vida }\end{array}$ & 0,95 \\
$\begin{array}{l}\text { Y: PPTT } \\
\text { X: Satisfacción por la } \\
\text { vida }\end{array}$ & \\
$\begin{array}{l}\text { Y:Autoestima (baja- } \\
\text { media) }\end{array}$ & \\
$\begin{array}{l}\text { X: Satisfacción por la } \\
\text { vida }\end{array}$ & 0,95 \\
Y:Autoestima (alta) & $y=31,913 \ln x-1,5861$ & \\
\hline
\end{tabular}

\section{Correlaciones de las ADPs y los MA}

Los resultados muestran en general que existe una correlación positiva entre las puntuaciones de las diferentes ADPAs y los MA (Tabla $5, R^{2}=0,97-0,98$ ) por lo que el entrenamiento de cada una de ellas conseguirá una mejora en el afrontamiento de MA. 
Tabla 5. Correlaciones del afrontamiento de MA con las diferentes ADPAs.

\begin{tabular}{|c|c|c|c|}
\hline Variables & Tipo de Ecuación & Ecuación & $\mathrm{R}^{2}$ \\
\hline $\begin{array}{l}\text { Y: Afronta Miedo } 1 \\
\text { X: Área Social }\end{array}$ & Exponencial & $y=5,9343 e^{0,0742 x}$ & 0,98 \\
\hline $\begin{array}{l}\text { Y: Afronta Miedo } 2 \\
\text { X: Área Social }\end{array}$ & Exponencial & $y=5,7152 e^{0,0857 x}$ & 0,98 \\
\hline $\begin{array}{l}\text { Y: Afronta Miedo } 3 \\
\text { X: Área Social }\end{array}$ & Potencial & $y=1,9858 x^{0,8526}$ & 0,98 \\
\hline $\begin{array}{l}\text { Y: Afronta Miedo } 1 \\
\text { X: Área Moral }\end{array}$ & Exponencial & $y=6,7211 e^{0,057 x}$ & 0,97 \\
\hline $\begin{array}{l}\text { Y: Afronta Miedo } 2 \\
\text { X: Área Moral }\end{array}$ & Exponencial & $y=5,626 e^{0,0782 x}$ & 0,97 \\
\hline $\begin{array}{l}\text { Y: Afronta Miedo } 3 \\
\text { X: Área Moral }\end{array}$ & Logarítmica & $y=10,873 \ln (x)-11,38$ & 0,98 \\
\hline $\begin{array}{l}\text { Y: Afronta Miedo } 1 \\
\text { X: Área Emocional }\end{array}$ & Lineal & $y=0,8853 x+3,3492$ & 0,97 \\
\hline $\begin{array}{l}\text { Y: Afronta Miedo } 2 \\
\text { X: Área Emocional }\end{array}$ & Lineal & $y=1,148 x+2,4001$ & 0,97 \\
\hline $\begin{array}{l}\text { Y: Afronta Miedo } 3 \\
\text { X: Área Emocional }\end{array}$ & Lineal & $y=1,4073 x-0,1544$ & 0,98 \\
\hline $\begin{array}{l}\text { Y: Afronta Miedo } 1 \\
\text { X: Área Cognitiva }\end{array}$ & Lineal & $y=0,8715 x+4,2464$ & 0,98 \\
\hline $\begin{array}{l}\text { Y: Afronta Miedo } 2 \\
\text { X: Área Cognitiva }\end{array}$ & Lineal & $y=1,1132 x+2,5806$ & 0,97 \\
\hline $\begin{array}{l}\text { Y: Afronta Miedo } 3 \\
\text { X: Área Cognitiva }\end{array}$ & Exponencial & $y=6,284 e^{0,0793 x}$ & 0,98 \\
\hline
\end{tabular}

Sin embargo, en las correlaciones analizadas entre las puntuaciones de afrontamiento de MA y de autoestima se obtuvo que el género no es concluyente sino más bien el nivel de autoestima, obteniendo diferentes correlaciones para las diferentes áreas según el nivel de autoestima de la muestra (Tabla 6).

Tabla 6. Correlaciones de la autoestima con las diferentes ADPAs.

\begin{tabular}{lccc}
\hline Variables & Tipo de Ecuación & Ecuación & $\mathrm{R}^{2}$ \\
\hline $\begin{array}{l}\text { X: Autoestima baja- } \\
\text { media }\end{array}$ & Exponencial & $y=3,5935 e^{0,0474 x}$ & 0,96 \\
$\begin{array}{l}\text { Y: Miedo 1 } \\
\text { X: Autoestima alta }\end{array}$ & Exponencial & $y=3,921 e^{0,0243 x}$ & 0,98 \\
$\begin{array}{l}\text { Y: Miedo 1 } \\
\text { X: Autoestima baja- } \\
\text { media }\end{array}$ & Logarítmica & $y=-12,66 \ln (x)+54,91$ & 0,96 \\
$\begin{array}{l}\text { Y: Miedo 2 } \\
\text { X: Autoestima alta }\end{array}$ & No correlaciona & & \\
$\begin{array}{l}\text { Y: Miedo 2 } \\
\text { X: Autoestima baja }\end{array}$ & Lineal & $y=-1,4788 x+50,813$ & 0,97 \\
$\begin{array}{l}\text { Y: Miedo 3 } \\
\text { X: Autoestima media }\end{array}$ & Lineal & $y=0,7917 x-8,5833$ & 0,96 \\
$\begin{array}{l}\text { Y: Miedo 3 } \\
\text { X: Autoestima alta }\end{array}$ & Lineal & $y=0,3596 x+1,6101$ & 0,97 \\
Y: Miedo 3 & & & \\
\hline
\end{tabular}


En concreto, todas correlacionaron excepto para el par: puntuaciones de autoestima elevada-puntuaciones de afrontamiento del miedo a lo desconocido. Además, todas las correlaciones fueron positivas (un aumento de la autoestima mejora el afrontamiento de MA) excepto en el caso de los subgrupos de autoestima baja del miedo 2 y del miedo 3 donde un incremento de la autoestima empeora la capacidad para afrontar el miedo al fracaso 0 a lo desconocido. En este sentido, sería interesante que el modelo usado describiera no sólo el autoconcepto y la autoestima como ejes principales del desarrollo positivo personal sino otros activos como la autonomía, el sentido de pertenencia o el autocontrol. Sería importante pues conocer si existe relación de estos otros activos con el afrontamiento de MA y si es así trabajarlos en las aulas junto con la autoestima. Por tanto, se acepta que existe relación entre autoestima y afrontamiento de MA, para todos los casos anteriores excepto para el subgrupo de autoestima alta y Miedo 2 (a lo desconocido).

\section{CONCLUSIONES}

El test PPT evalúa efectivamente las ADPAs (Alfa de Cronbach =0,7-0,9) a los alumnos y les informa sobre sus valores para que conozcan sus propias debilidades. En este caso, la muestra analizada goza de cierta salud psico-social aunque mejorable. En concreto, las capacidades de creatividad y toma de decisiones y optimismo y tolerancia a la frustración no sólo se encontraban por debajo de la media general, sino que fueron las capacidades con más asimetría positiva dado, probablemente, el efecto que ha tenido la COVID-19 sobre ellas, por lo que sería interesante trabajarlas a nivel de clase. Esto podría mejorar la correlación de la autoestima con el afrontamiento del miedo a lo desconocido (con valores muy atípicos).

Finalmente, en este trabajo, se ha tenido en cuenta variables como el género o nivel de autoestima; pero sería interesante además recoger otras variables como: contacto directo con personas con COVID-19 o muerte cercana por COVID-19 y ver cómo está afectando la COVID-19 a las ADPA.

\section{REFERENCIAS BIBLIOGRÁFICAS}

Beltrán-Martínez, S. (2014). Los miedos en la pre-adolescencia y adolescencia y su relación con la autoestima: Un análisis por edad y sexo. Revista de Psicología Clínica con Niños y Adolescentes, 1(1), $27-36$.

Benson, P.L., Mannes, M., Pittman, K. y Ferber, T (2004). Youth development, developmental assets and public policy. En R. Lerner \& L. Steinberg (Eds.), Handbook of adolescent psychology (2ª ed., pp. 781-814). Nueva York: John Wiley.

Berasategi Sancho, N. (2020) Resultados En Idoiaga Mondragon, N., Dosil Santamaría, M., Picaza Gorrochategui, M., y Ozamiz Etxebarria, N. (Eds). Las voces de los niños y de las niñas en situación de confinamiento por el COVID-19. (pp. 11-30) Disponible en: https://web-argitalpena.adm.ehu.es/pdf/ USP00202291.pdf

Bleidorn, W., Arslan, R. C., Denissen, J. J., Rentfrow, P. J., Gebauer, J. E., Potter, J., y Gosling, S. D. (2016). Age and gender differences in self-esteem-A cross-cultural window. Journal of personality and social psychology, $111(3), 396$.

Byrne, B. (2000). Relationships between anxiety, fear, self-esteem, and coping strategies in adolescence. Adolescence, 35(137), 201-215.

Campo-Arias, A. y Oviedo, H. C. (2008). Propiedades psicométricas de una escala: la consistencia interna. Revista de salud pública, 10, 831-839.

Fernández-Zabala, A., Goñi, E., Rodríguez-Fernández, A. y Goñi, A. (2015). Un nuevo cuestionario en castellano con escalas de las dimensiones del autoconcepto. Revista Mexicana de Psicología, 32(2), 149-159.

Fuentes, M. C., García, J. F., Gracia, E. y Lila, M. (2011). Autoconcepto y ajuste psicosocial en la adolescencia. Psicothema, 23(1), 7-12.

García, F., y Musitu, G. (2014). Manual AF-5: Autoconcepto Forma-5. Madrid: TEA Ediciones.

Gómez-Becerra, I., Contreras, J. M. F., Romero, M. P. A., López, P. S. y Torres, M. F. (2020). Evolución del estado psicológico y el miedo en la infancia y adolescencia durante el confinamiento por la COVID-19. Revista de 
Psicología Clínica con Niños y Adolescentes, 7(3), 11-18.

Góngora, V., Fernández, M. y Castro, A. (2012). Estudio de validación de la escala de autoestima de Rosenberg en población adolescente de la ciudad de Buenos Aires. Perspectivas en Psicología, 7 (1), 24-30.

Gordillo, M. G., Castro, F. V., Herrera, S. S., Acuñas, M. G. y Solanes, T. G. (2011). Diferencias en el autoconcepto de adolescentes extremeños en función del género, el nivel socioeconómico-cultural y la población de pertenencia. Revista de Educación Campo Abierto, 30 (2), 65-78

Huebner, E. S. (1991). Further validation of the Students Life Satisfaction Scale: The independence of satisfaction and affect rating. Journal of Psychoeducational Assessment, 9, 363-368.

Huremovic, D. (2019). Psychiatry of pandemics: a mental health response to infection outbreak. New York: Springer

Lerner, R. M., Lerner, J. V., Almerigi, J., Theokas, C., Phelps, E., Gestsdottir, S., Naudeau, S., Jelicic, H., Alberts, A. E., Ma, L., Smith, L. M., Bobek, D. L., Richman-raphael, D., Simpson, I., Christiansen, E. D. ; Von Eye, A (2005). Positive youth development, participation in community youth development programs, and community contributions of fifth grade adolescents: Findingsfrom the first wave of the 4-H Study of Positive Youth Development. Journal of Early Adolescence, 25, 17-71

Méndez, F. X., Inglés, C. J., Hidalgo, M. D., García-Fernández, J. M., y Quiles, M. J. (2003). Los miedos en la infancia y la adolescencia: un estudio descriptivo. Revista Electrónica de Motivación y Emoción, 6(13), $150-$ 163.

Musitu, G., Román, J. M. y García, E. (1988). Familia y educación: prácticas educativas de los padres y socialización de los hijos. Barcelona: Labor.

Oliva Delgado, A., Hernando Gómez, Á., Parra Jiménez, Á., Pertegal Vega, M. Á., Ríos Bermúdez, M., y Antolín Suárez, L. (2008). La promoción del desarrollo adolescente: recursos y estrategias de intervención. [s.l.]:Junta de Andalucía, Consejería de Salud.

Padilla, M. T., García, S. y Suárez, M. (2010). Diferencias de género en el autoconcepto general y académico de estudiantes de $4^{\text {a }}$ de ESO. Revista de educación, 352, 495-515.

Pérez Grande, M. D. (2000). El miedo y sus trastornos en la infancia. Prevención e intervención educativa.

Piers, E. V. y Harris, D.B. (1969). The Piers-Harris Children's Self-Concept Scale. Nashville, Tennesse: Counselor Recording and Tests.

Rodríguez, A., Droguett, L. y Revuelta, L. (2012). Ajuste escolar y personal en la adolescencia: el papel del autoconcepto académico y del apoyo social percibido. Revista de Psicodidáctica, 17 (2), 397-414.

Roth, J. L. y Brooks-Gunn, J. (2003). What exactly is a youth development program? Answers from research and practice. Applied developmental science, 7(2), 94-111.

Rosenberg, M. (1965). Society and Adolescent self-image. Princeton: University Press.

Serrano Muñoz, A. M. (2014). Diseño y validación de un cuestionario para medir la autoestima infantil. La relación entre autoestima, rendimiento académico y las variables sociodemográficas. (Tesis doctoral inédita) Universidad de Córdoba. Recuperado de https://helvia.uco.es/xmlui/bitstream/handle/10396/11580/20140 00000900.pdf

Tapia (2016) La influencia que los estereotipos de género ejercen sobre el autoconcepto y la autoestima Tesis de pregrado. Recuperado de: https://uma.academia.edu/CarolinaTapia.

Villar, I., Michael, W. y Gribbons, B. (1995). The development and construct validation of a Portuguese version of an academic self-concept scale. Educational and Psychological Measurement, 55 (1), 115-123.

Zubeidat, I., Salinas, J. M., y Sierra, J. C. (2007). Escala de Miedo a la Evaluación Negativa y Escala de Evitación y Malestar Social: fiabilidad y validez en una muestra de adolescentes españoles. Clínica y Salud, 18(1), 5781. 
APLICACIÓN DEL CUESTIONARIO "POSITIVE_PERSONAL_TOOL” Y SU RELACIÓN CON LOS MIEDOS ADOLESCENTES

\section{AGRADECIMIENTOS}

Mi más sincero agradecimiento al equipo de orientación y dirección de los dos institutos encuestados, así como a Maria del Carmen Canto López y Maria Dolores Huertas personal docente investigador de la Universidad de Cádiz. 